\title{
DATA MONITORING AND INTERIM ANALYSES IN THE PHARMACEUTICAL INDUSTRY: ETHICAL AND LOGISTICAL CONSIDERATIONS
}

\author{
FRANK W. ROCKHOLD \\ SmithKline Beecham Pharmaceuticals, Four Falls Corporate Center, Route 23 \& Woodmont Avenue, P. O. Box 1510, King \\ of Prussia, PA 19406, U.S.A. \\ AND \\ GREGORY G. ENAS \\ Lilly Research Laboratories, Lilly Corporate Center, Indianapolis, IN 46285, U.S.A.
}

\begin{abstract}
SUMMARY
The characteristics of data monitoring and the need for the use of data monitoring committees in clinical trials sponsored by the pharmaceutical industry differ from those of trials sponsored by government. Data monitoring is a continuous process in industry trials due to the regulatory requirements and the need to more thoroughly evaluate safety of new compounds. As part of this process, interim analyses are employed to make decisions about treatment effects. In some cases, such analyses may require the use of an external data monitoring committee to assist in the data review, analysis and decision making. A number of examples of interim analyses, with and without data monitoring committees, are discussed. Issues surrounding the need for external data monitoring committees and recommendations are presented. In particular the issues of sponsor participation in the data monitoring committee and controls of the decision making process are considered.
\end{abstract}

\section{INTRODUCTION}

This paper presents some of the approaches the pharmaceutical industry has taken to issues related to data monitoring and interim analyses and in particular the use of external data monitoring committees. In our current responsibilities in biometrics we are primarily involved in all phases of the interim analysis process but also have managed many other aspects of the clinical trial process including monitoring, safety and clinical data management. Therefore the examples and views are presented from a broad perspective. There a number of ramifications of the data monitoring and the interim analysis process in trials that are sponsored by our industry. Many of these are very similar to the problems faced by the National Institutes of Health, but some are very different. Within one sponsor company alone hundreds of trials are implemented every year, from phase I to phase IV, on $\mathbf{4 0}$ or more projects simultaneously. Our trials have from 10 to 10,000 patients. Each patient has a case report form which ranges from 25 to 200 pages. Thus our internal trials generate a tremendous volume of work. We also fund a number of external trials for which data responsibilities are transferred. Most of our work focused on registering a product. Many of our trials are run for the express purpose of generating publications, however, while others represent purely basic research.

0277-6715/93/050471-09\$09.50

(C) 1993 by John Wiley \& Sons, Ltd. 
For purposes of clarity, working definitions of monitoring and interim analyses are outlined below. In the pharmaceutical industry we view data monitoring and interim analyses as two distinct processes, and therefore it is important to provide a definition of these terms.

Data monitoring: 'The process of scrutinizing logistics of a clinical trial so that the intended plan of the trial is realized. Activities associated with monitoring include all issues related to protocol compliance. Issues related to patient safety are also addressed using explicit protocol defined decision rules.

This is clearly a patient level activity which is performed by a number of individuals within the company as will be covered later.

As for interim analyses we work with the following definition: ${ }^{1}$

The process of scientific inference and decision making throughout the course of the clinical trial. It is understood that all decisions not stipulated $a$ priori in the protocol are the results of interim analyses. All inferences regarding efficacy and some safety variables are defined in this framework.

This is clearly a group level inferential activity. All such analyses must be carefully controlled and planned to protect the integrity of the trial, but, as will be discussed later, there are many issues beyond the statistical ones that affect the ability to perform such analyses.

Other definitions have been proposed, ${ }^{2}$ but are not as specific to clinical trials in the pharmaceutical industry.

\section{CHARACTERISTICS OF INDUSTRY-SPONSORED TRIALS}

There are some characteristics of industry trials which may be somewhat different from government-sponsored trials or at least approached from a different perspective. There are many other differences between industry and government-sponsored trials but these are the salient points for this discussion.

Since the sponsor in pharmaceutical industry trials most often acts as the co-ordinating center, that is, performs all the data review and clinical data management, rarely is there a need for an external co-ordinating centre. Exceptions to this would, of course, be studies that are contracted with an independent vendor. In the process of data review, the clinical research associate, the physician, nurses, and clinical data management are all blinded with respect to treatment as they review the data.

There is, of course, extensive Food and Drug Administration (or other regulatory agency) interaction and involvement in trial design. This scrutiny is often very helpful because the regulatory agency sees a far greater number of trials in a particular therapeutic area than the company itself would be exposed to. Many times the benefit of that experience results in the design of a better trial.

We are also bound by a number of regulatory reporting requirements relating to serious adverse events, investigational new drug updates, as well as other regulations. There are groups within the company that are dedicated to nothing other than ensuring that these requirements are met.

One aspect of trial analysis that is somewhat different in the pharmaceutical industry is that by far and away the largest amount of effort put in the analysis of a trial is expended on the safety data. This is partly due to regulatory requirements, partly due to the need to generate proper labelling for new chemical entities, and also to generate periodic management summaries for internal consumption. 
As mentioned above, one of our primary goals is to seek a regulatory submission as opposed to solely a publication, which may be the primary interest of trials sponsored by other institutions. Our ultimate goal is to establish an appropriate benefit-to-risk ratio so that the drug can be properly labelled for marketing.

It is also important to note that many of the trials that we perform are smaller than public health service trials and many involve non-life-threatening endpoints. Even though not all the trials involve mortality as an endpoint, they are not necessarily any less important from a public health viewpoint; arthritis, anti-infective, and psychotropic therapies, for example, contribute very heavily to improving the health of the population.

As a pharmaceutical company marketing a product, we are also responsible for reporting events in future patients who consume our products as well as those individuals who participated in our trials. We therefore continue to monitor the results and events that occur even in patients who are consuming the drug outside of the clinical trial process. Thus, it is incumbent upon us to evaluate even more thoroughly relevant trial safety data.

\section{ETHICAL PRIORITIES IN THE CONDUCT OF TRIALS}

Our top priority in the execution of clinical trials is the clinical trial patient. We must ensure they have the most up-to-date information, the best available care, and the best monitoring that we as sponsors can provide.

The next priority is the investigator who is treating the patient. Since we are relying on the investigators to provide the best available medical care, we must ensure that they have all the relevant information and support that is available.

We also have a responsibility to future patients who will ultimately consume the medication being studied, assuming it is approved for marketing.

The last priority on this list is the institutional objective. Since our institutional objective is to make a profit and to pay dividends to shareholders, we feel this objective will always be achieved as long as the first three that we have outlined are met. These principles have a definitive impact on the approach to data monitoring and interim analyses.

\section{INDUSTRY DATA MONITORING PRACTICES}

It is instructive to review characteristics of data monitoring within a pharmaceutical company as they are somewhat different from what is done within various government agencies. There are at least three levels of a case report review form before it is even entered into computer data bases.

Reviews are always performed by the field staff at the site as well as in-house nurses and physicians. This is done according to regulations, good clinical practices, and corporate standard operating procedures. There is also an ongoing data management review that starts with the monitoring and internal review and includes queries going back out to the field. A.ll individuals examining data are blinded to treatment assignment. Individual patient codes are rarely if ever unblinded in a trial except in the extreme case of serious adverse events that must be reported immediately to the government. Among the responsibilities are the 3 and 10 day reporting requirements to which we must adhere. Internal safety groups are responsible for maintaining the reporting requirements. If necessary, the code can be broken for an individual patient for regulatory reporting purposes without anyone directly involved in the trial having to be aware of it.

The legal liability of the company physician in the pharmaceutical drug development process requires that he or she be intimately involved with the running of a clinical trial and the 
monitoring of patient safety on a continuous basis. For these reasons and because company sponsors normally act as their own co-ordinating centre, most trials can be executed without the need for an external data monitoring board. There may be situations, as will be discussed later, where trials are run by external agencies, for example in critically ill patients or possibly in trials where the endpoint under study is severe enough that there may be an ethical obligation to have an independent review board.

In summary, the data monitoring function in a pharmaceutical industry clinical research department is a continuous exercise because of the various ethical, regulatory and data management issues that have been outlined. These functions are performed on every trial that we run.

\section{REASONS FOR PERFORMING INTERIM ANALYSES}

In contrast to data monitoring, however, we would not always choose to perform interim analyses on our trials. Some situations where we would choose to do so are outlined below.

We may choose to perform an interim analysis for a number of reasons related to ethical issues. As mentioned above, if the endpoint is severe enough, or the results dramatic enough, one would want to consider terminating a trial for efficacy reasons, although the primary focus of many of our trials is safety and in many cases even if efficacy has been proven, the trials are mandated to run for longer periods of time in order to properly evaluate patient safety.

We may also perform interim analyses with regard to ethical issues from a safety standpoint. As indicated above, however, there is an ongoing safety review of each individual patient as the data come into the company. While the treatment assignment is not known by the individual reviewing the documents, one would develop some concern if the incidence of a particular event was higher than otherwise anticipated. One would certainly include safety analyses as part of any interim analysis process.

There have been a number of instances where a regulatory agency in a given country has requested information on the safety or efficacy of a compound. It may be that the only appropriate trial available to answer the inquiry is one ongoing somewhere else in the world. It may therefore be necessary to perform an interim analysis in order to respond to the regulatory agency's question.

Occasionally, although rarely, an interim analysis may be specified in the protocol in order to extract information for planning purposes. For example, one may be trying to plan for production of supplies for future trials by looking at analyses of dose, or to get an indication of the likelihood of having to build more manufacturing sites in order to produce material. Finally, it may be of interest to do an interim analysis on a compound if information from other studies indicates the compound may not be working as effectively as possible and there is a possibility we can terminate work on an ineffective drug, saving patients, time and resources.

All of the above are reasons why an interim analysis may be planned for in a protocol, primarily conducted on a few, pre-specified occasions. Reasons for considering only a few interim analyses include one's ability to protect the scientific integrity of the trial, as well as the purely practical and logistical reasons that relate to timing and availability of resources and processing of data.

\section{LOGISTICAL ISSUES IN INTERIM ANALYSES}

Some of the logistical issues that need to be taken into consideration in performing interim analyses in our research environment are the timing of the analysis in relation to the start and end 
of the trial, as well as the timing of the interim analysis in relation to other activities ongoing in the company. Some of these issues are similar to those for National Institutes of Health sponsored trials, but they provide the necessary background for our need for interim analyses and/or data monitoring committees.

The speed of the analysis is a critical element that is not always adequately considered. Because of the lag time between the enrolment of the necessary number of patients and the production of the actual interim analysis, it is critical that as little time as possible be spent analysing the data for the sake of the patients who continue to be enrolled in the trial. It can be a difficult analytical problem to account for the patients who have enrolled in a study after the cutoff for the interim analysis. This is particularly a problem in chronic disease treatment trials as opposed to prevention trials.

It often requires almost as many resources to perform an interim analysis as it does to perform the final analysis of a trial. Many times we have to alter our field data retrieval process to acquire the necessary data and co-ordinate the timings appropriately. This is caused by a number of factors, not the least of which is the fact that many of our trials have a short-term efficacy phase followed by long-term safety extensions, so that while the patients' efficacy data may come in-house to be analysed, the patients will stay on the study for much longer periods of time and continue to accrue data.

It is inevitable in the interim analysis process that we make decisions on data quality that we are not faced with in the final analysis. Most of this is due to the urgency of wanting to proceed with the analysis and the fact that the interim analysis often involves a subset and not all of the data being collected.

In the pharmaceutical industry there are regulatory constraints with regard to performing interim analyses. It is not that we are prohibited from doing such analyses, but we must very carefully document all interim analyses and whatever effects they may have on the final conclusion. If the interim analysis does not result in the termination of the trial then we must clearly document what effects, if any, this may have on the ultimate inference to be made and therefore the labelling for the new product. This is no different from a standard scientific approach, but we are directly accountable to the regulatory agencies.

\section{EXAMPLES}

A number of examples of industry-sponsored trials that involved interim analyses are presented below. Some of these involve scheduled interim analyses, some unscheduled. Some of them involved external data monitoring committees, and some of them involved internal data monitoring groups.

\section{Example 1: Congestive Heart Failure Trial}

The first example is a placebo-controlled trial that enrolled patients with congestive heart failure to test the efficacy of an inotropic agent, using exercise tolerance as an endpoint. ${ }^{3}$ An interim analysis was planned by internal staff to be performed after half of the patients had been enrolled. However, because the medical monitor was carefully reviewing the data as it was coming in and communicating with the sites appropriately, he detected a higher than expected mortality rate in the group as a whole.

After consultation with the investigators and a number of outside consultants, the decision was made to perform an unscheduled interim analysis. Because there was an interim analysis plan built into the protocol, the rule was adapted to perform the analysis. Although not statistically significant, the mortality rate was higher on the test drug than control. 
Even though the difference was not significant and the external experts recommended continuation of the trial, the company decision was to terminate it. While having a data monitoring board in this example might have provided some additional organization to the process, it is unlikely the problem would have been identified or resolved any sooner. In the end the decision to terminate the trial was clearly in the best interest of everyone involved.

\section{Example 2: Prevention of upper gastrointestinal bleeding in intensive care unit patients}

This was a study comparing the efficacy of cimetidine versus placebo in the prophylaxis of stress-related upper gastrointestinal bleeding in critically ill patients in intensive care units. ${ }^{4}$ The trial was planed to enrol 200 patients. Because the treatment being employed had been available for many years for use in other indications, it was already being employed to a large extent in this population of patients. However, since it had never been proven effective for this indication, the Food and Drug Administration required the sponsor to perform a placebo-controlled trial. In order to recruit the requisite number of investigators, a large number of institutions were approached and many investigators agreed to participate only after they realized an interim analysis was planned. Owing to the ethical issues and medical importance of the trial it was decided to assemble an external data monitoring board to perform the interim analysis. This analysis had very clearly defined decision rules. After performing the interim analysis, the decision was made to stop the trial based on overwhelming efficacy and no safety issues.

As mentioned above, there is often a problem of what to do with patients who are enrolled while the analysis is being conducted. This analysis was conducted in an extremely rapid manner and yet 31 patients were enrolled while the analysis was being performed. In this case, the results were no different when those 31 patients were added in, but this can be a difficult issue to deal with.

The data monitoring committee consisted only of individuals external to the company. One statistician and three physicians were involved. All of the data processing support for the interim analysis was performed internally, and a sealed copy of the code was sent by the internal safety department to the data monitoring committee.

\section{Example 3: APSAC Intervention Mortality Study (AIMS Trial)}

This was a placebo-controlled trial to evaluate the effect of anisoylated plasminogen streptokinase activator complex (APSAC) (or Eminase) on mortality in the post-myocardial infarction population. ${ }^{5}$ The trial was designed to enrol 2000 patients. It had an external steering committee and data monitoring board as well as an independent statistician to analyze the mortality results. An interim analysis was to be performed after 1000 patients; after results had come in from other trials, however, the plan was altered to do an analysis after every 500 patients.

The trial was terminated after 1004 patients were enrolled due to overwhelming evidence of an improvement in mortality with the drug. This trial ultimately served as the primary piece of information which allowed Eminase to be marketed in most countries around the world.

The data monitoring committee received unblinded interim results at predefined intervals to ensure that patients were not being adversely affected by the therapy. The data monitoring committee consisted of two physicians and one statistician.

\section{Example 4: Alzheimer's disease trial}

This was a trial comparing the effect of an experimental therapy to placebo on the Alzheimer's Disease Assessment Scale in patients with Alzheimer's disease. The trial had no pre-planned 
interim analysis and no external data monitoring committee. After data from other trials on the same therapy became available, however, it became clear that an interim analysis would be necessary, particularly given the nature of the disease under study. The protocol was formally amended and submitted to the Food and Drug Administration with a well-defined decision rule for interim analyses. Because the trial was placebo-controlled, the primary concern was to be able to terminate the trial early if no effect was being observed. A decision rule developed by Wieand and Thernau in $1987^{6}$ was employed.

The interim analysis indicated that there was no reason to continue the trial based solely on efficacy; because the trial had been widely publicized, however, the enrolment had been so rapid that by the time the interim analysis was completed the planned number of patients had already been enrolled. This happened because the patients were required to have 3 to 6 month follow-up data in order to be included in the efficacy analysis, and during that lag time the remaining patients were enrolled. This is an excellent example of 'enrolment lag time' mentioned earlier as a potential problem, particularly in chronic treatment trials.

It is unclear what the interim analysis may have achieved in this situation. As soon as the decision was made to terminate the trial, even though all patients had been enrolled, the investigators were immediately informed and the patients' therapy was withdrawn. Whether an external data monitoring board would have been helpful in this situation is questionable. A major difficulty was that the case report form was not designed with interim analysis in mind; the performance of this analysis therefore created a tremendous burden both on the field monitoring staff and on the in-house data management staff. For exercises such as this, the monitoring and data management practices need to be considered in the planning and organization of the trial.

\section{Example 5: European Myocardial Infarction Project (EMIP) Trial}

The last example is the European Myocardial Infarction Project (EMIP), a trial involving 10,000 patients to study the use of thrombolytic therapy in an ambulance. ${ }^{7}$ Mortality was the primary endpoint. The study was contracted to an outside organization with an external policy board and oversight by an external project management group.

The protocol specified that interim analyses would be performed after each group of 2500 patients were enrolled. Owing to the size of the trial, the seriousness of the endpoint, and the fact that it was conducted totally independently of the company, both a co-ordinating centre and a data monitoring board were critical to the execution of this trial. This same process would be recommended for trials like this in the future.

\section{ISSUES AND RECOMMENDATIONS FOR DATA MONITORING COMMITTEES IN INDUSTRY TRIALS}

External data monitoring boards might be recommended for use in trials where there is the potential for severe adverse events or if a mortality or life-threatening endpoint is being studied. This allows an extra margin of safety and extra pairs of eyes helping the sponsor do their job. Likewise one might consider using an external data monitoring board for planned interim analyses or when the information may be used for regulatory purposes. This might help convince regulatory agencies that the trial was conducted in a completely objective manner.

One might also recommend the use of such external committees when the study is contracted out by the sponsor and therefore company standard operating procedures may not be implemented. Data processing and other operational tasks are probably most often going to be supplied from the pharmaceutical company. Since we are responsible for maintaining an ongoing 
worldwide data base for all of our products it is much easier for us to maintain that in-house. This, of course, adds another layer of communication in dealing with an external data monitoring board, since we do not normally have a co-ordinating centre external to the company.

If a choice is made to use an external data monitoring committee, it is clear that prior to the start of the trial one must have a very good understanding of who has control of the decision process. For instance, should the data monitoring committee be able to stop the trial at any time? The answer to that is probably yes. Should the sponsor be able to stop the trial at any time? The answer to that is probably yes as well.

Another question that has been asked in various institutions is: should the sponsor be represented on the data monitoring board? There are arguments for and against this. Individuals from the sponsor will have a much better knowledge of the drug and its pharmacologic actions as well as its overall safety profile in order to put results in context. It could be argued, however, that individuals from the sponsor would have a vested interest in the results of the trial and therefore would potentially impinge on the independence of the data monitoring board. If it is best to use an independent data monitoring committee in some situations, the preference is to keep it just that - independent.

\section{CONCLUSIONS}

In conclusion, adequate data monitoring must be and is performed to protect patient welfare. This could most often be done in trials sponsored by in the pharmaceutical industry without the use of data monitoring boards.

External data monitoring boards might, however, be very useful for a subset of industrysponsored trials, in particular those in which interim analyses are being performed for the purpose of stopping early for efficacy. As has been outlined, interim analyses may be performed in industry-sponsored trials for different reasons than those in government- or academic-sponsored trials. This is due partially to the regulatory and commercial environment and also to the corporate legal responsibility to perform ongoing follow-up for all of our trials. Thus, even though we might employ an external data monitoring board it is certainly not as integral a part of all the trials we perform as it would be in the National Institutes of Health environment.

While this paper raises a number of question it also gives some insight into how a pharmaceutical company would approach the prospect of doing interim analysis, the reasons for such analysis, and some of the logistical issues in using an external data monitoring board for industry trials.

ACKNOWLEDGEMENT

We would like to thank Susan Cantz for her outstanding efforts in helping to prepare this manuscript.

\section{REFERENCES}

1. Enas, G. G., Rockhold, F. W., Dornseif, B. E., Sampson, C. B. and Wuu, J. 'Monitoring and interim analysis of clinical trials: A perspective from the pharmaceutical industry', Controlled Clinical Trials, 10(1), 57-70 (1989).

2. Armitage, P. 'Interim analysis in clinical trials', Statistics in Medicine, 10, 925-937 (1991).

3. Rockhold, F. W. and Enas, G. G. 'Practical approaches to the design and conduct of interim analyses', in Peace, K. E. (ed.), Biopharmaceutical Sequential Statistical Applications, Marcel Dekker, 1992, Chapter 1.2.

4. Martin, L. F., McL. Booth, F. V., Karlstadt, R. G., Silverstein, J. H., Jacobs, D., Hampsey, J., Bowman, S. C., D'Ambrosio, C. A. and Rockhold, F. W. 'Continuous intravenous cimetidine decreased stress-related upper gastrointestinal hemorrhage without promoting pneumonia', Critical Care Medicine, 21(1), 19-30 (1993). 
5. APSAC Intervention Mortality Study Trial Study Group. 'Effect of intravenous APSAC on mortality after acute myocardial infarction: Preliminary report of a placebo-controlled clinical trial', The Lancet, 1 , 545-549 (1988).

6. Wieand, S. and Therneau, T. 'A two-stage design for randomized trials with binary outcomes', Controlled Clinical Trials, 8(1), 20-28 (1987).

7. European Myocardial Infarction Project. 'Potential time saving with pre-hospital intervention in acute myocardial infarction', European Heart Journal, 9(2), 118-124 (1988).

8. Castaigne, A. D., Herve, Ch., Duval-Moulin, A. M., Gaillard, M., Dubois-Rande, J. L. and Lellouche, D. 'Pre-hospital thrombolysis, is it useful?', European Heart Journal, 11, (Suppl. F), 43-47 (1990). 